\title{
Resource Allocation Using Dynamic Spreading Gain Control for Wideband CDMA Networks Supporting Multimedia Traffic
}

\author{
Hailong Huang and Francois Chin \\ Department of Electrical \& Computer Engineering, National University of Singapore, 10 Kent \\ Ridge Crescent, Singapore, 119260 engp0921@nus.edu.sg
}

Institution of Communication Research, ,20 Science Park Road, \#02-34/37, Singapore Science Park II, Singapore, 117674 chinfrancois@cwc.nus.edu.sg

\begin{abstract}
In this paper, a resource allocation scheme, Dynamic Power and Spreading Gain Control (DPGC), for voice and data integrated wideband CDMA networks is proposed to achieve multiple QoS requirements. Specifically, using fixed SIR and spreading gain for voice users, both power utility function and the packet throughput are maximized for each data user by adjusting spreading gain and the target signal-to-interference ratio (SIR) in the basestation receiver. Computer simulation has demonstrated that the proposed resource allocation scheme DPGC is effective in increasing throughput of data users with given SIR requirement and power constraint.
\end{abstract}

Key words: Spreading Gain Control, Wideband CDMA, Multimedia

\section{INTRODUCTION}

In a voice and data integrated wideband CDMA wireless network, both the power of all the users and transmission rates of data users may be considered as controllable resources. To cater for the requirement of high speed communication especially data applications in 3G wireless networks, we have to maximize data users' packet data rate /throughput while still stick to tight power limits due to battery size. Typically, the quality of service

The original version of this chapter was revised: The copyright line was incorrect. This has been
corrected. The Erratum to this chapter is available at DOI: 10.1007/978-0-387-35618-1_37 
(QoS) in wideband CDMA can be controlled by an appropriate selection of transmitted power [1] and spreading gain [2].

In the previous papers, the different power control (PC) algorithms fall into mainly two categories: those based on the measured values of received power (signal-level-based PC [3][4]); and those based on the measured signal-to-interference ratio (SIR-based PC, CIR balancing PC [5]). In this paper, we only consider the SIR-based PC because it can provide larger capacity and better grade of service. To make it applicable for data applications in $3 \mathrm{G}$ wireless systems that require dynamic high throughput (bit rate), we try to maximize data user's power utility function [6] through network assisted distributed SIR-based power control [7][8] in uplink and simultaneously keep voice user's power consumption at a level such that it can support the voice user's QoS requirement.

The basic idea of spreading gain control is to (in)decrease the spreading gain of data terminals as the MAI level (in)decreases, which can result in great improvements in data traffic throughput compared to fixed spreading gain [9]. The general issue we wish to address in this paper is how to assign powers and spreading gains to different classes of traffic (voice and data) so as to maximize information rate given a fixed number of users and QoS constraints. The QoS performance measure for voice traffic is the target bit error rate (BER), while the data traffic the minimum throughput requirement.

\section{SYSTEM MODEL AND PERFORMANCE MEASURES}

We now specify a system model for the wideband CDMA network and the corresponding performance measures for it. Assuming that there are two classes of traffic, voice and data, within a single cell, the aim is to determine the throughput performance for different number of voice and data users.

In this integrated wireless system considered, each user (voice and data) generates a sequence of data packets. And a new packet is generated as soon as the preceding packet is successfully delivered. Since the users continuously transmit packets, the number of active users in the system therefore equals to the users in the system (no inactive users). The model for voice users differs from the model for data users in that voice users do not retransmit packs with errors. The same packet length and coding scheme are assumed for both classes. 
The system channel rate is $R \mathrm{~b} / \mathrm{s}$, where $R=W / G$. W is the CDMA system chip rate and $G$ is the spreading gain. The DLC layer first converts (segment or combine) the original information packets and finally encodes them into the target-length packets using certain error detection coding. After that, the sequence of packets is of length $L$ bits with code rate $r(L r$ information bits). We assume that the probability of undetected transmission errors is negligible.

Given $N_{v}$ active voice users and $N_{d}$ active data users, the signal-tointerference ratio (SIR) for users in each class in the basestation receiver is defined as:

$$
\begin{aligned}
& S I R_{v}=\frac{P_{v} G_{v}}{\left(K_{v}-1\right) P_{v}+K_{d} P_{d}+\sigma^{2}} \\
& S I R_{d}=\frac{P_{d} G_{d}}{\left(K_{d}-1\right) P_{d}+K_{v} P_{v}+\sigma^{2}}
\end{aligned}
$$

where subscripts $v$ and $d$ indicate voice and data classes respectively. $P_{i}$ watts is the transmission power, and $\sigma^{2}$ is the noise power in the base station receiver. The power assignment $\left(P_{v}\right.$ and $\left.P_{d}\right)$ can mutually change $S I R_{v}$ and $S I R_{d}$ in a drastic way.

For voice users, since there is no need to retransmit the erroneous packets, we only concern about the BER and then assign a fixed spreading gain $G_{v}$.

$$
p_{b v}=k \exp \left(-\beta \gamma_{v}\right)
$$

where $k$ and $\beta$ are parameters which can be adjusted to match a particular coding scheme.

Data transmission is a sort of error-sensitive application, when the receiver detects an error in a packet, a selective ARQ protocol will request the source to retransmit the error packet again. Suppose the SIR is $\gamma_{d}$, the packet success rate at the receiver is:

$$
f\left(\gamma_{d}\right)=\left(1-p_{b d}\right)^{L r}=\left(1-k \exp \left(-\beta \gamma_{d}\right)\right)^{L r}
$$

Utility function for data traffic is defined as the number of information bits delivered accurately to a receiver for each joule of energy expended by the transmitter [10].

$$
U_{d}=\frac{L r f\left(\gamma_{d}\right)}{P_{d} L / R_{d}}=R_{d} r \frac{f\left(\gamma_{d}\right)}{P_{d}} \quad(\mathrm{~b} / \mathrm{j})
$$


where $\operatorname{Lrf}\left(\gamma_{d}\right)$ is the expected number of successfully transmitted information bits, and $P_{d} L / R_{d}$ means the energy consumed in transmitting one packet.

Now we define data throughput as the number of correctly received bits per second.

$$
T\left(\gamma_{d}\right)=\frac{\operatorname{Lrf}\left(\gamma_{d}\right)}{L / R_{d}}=W r f\left(\gamma_{d}\right) / G_{d}
$$

Since we adopt SIR balancing power control, all the active terminals have the same optimum targets, and then the same throughput.

\section{DYNAMIC POWER AND SPREADING GAIN CONTROL (DPGC) FOR MULTI-QOS WIDEBAND CDMA}

Now we describe the resource allocation scheme, DPGC, for this voice and data integrated wideband CDMA system. The resource allocation issue here is to select a proper assignment of powers and processing gains $P_{v}, P_{d}, G_{v}$ and $G_{d}$ so that $\mathrm{QoS}$ requirements are satisfied. The QoS measure for voice user is the BER, while that for data user is throughput. Now we aim to maximize the throughput of data users for fixed $N_{v}$ and $N_{d}$ in the capacity region, subject to an acceptable BER for voice users.

For voice users, since its data rate is very low, we assign a fixed spreading gain $G_{v}=256$ for them. If $\varepsilon_{v}$ is the maximum acceptable BER for voice user, we can get an equivalent constraint from Equation (3), i.e., the minimum target signal-to-interference ratio $\gamma_{v}^{*}$.

$$
S I R_{v} \geq \gamma_{v}^{*} \text { where } \gamma_{v}^{*}=\frac{1}{\beta} \ln \left(\frac{k}{\varepsilon_{v}}\right)
$$

The constraint (7) can be combined with (1) to obtain the following:

$P_{d} \leq A P_{v}-B$

where $A=\frac{G_{v}-\left(K_{v}-1\right) \gamma_{v}^{*}}{K_{d} \gamma_{v}^{*}}$ and $B=\frac{\sigma^{2}}{K_{d}}$

From (2), we can get the other equation:

$$
P_{d}=\frac{\gamma_{d}\left(K_{v} P_{v}+1\right)}{G_{d}-\left(K_{d}-1\right) \gamma_{d}}=C P_{v}+D
$$

where $C=\frac{K_{v} \gamma_{d}}{G_{d}-\left(K_{d}-1\right) \gamma_{d}}$ and 


$$
D=\frac{\sigma^{2} \gamma_{d}}{G_{d}-\left(K_{d}-1\right) \gamma_{d}}
$$

Then we can obtain the relationship between $P_{d}$ and $\gamma_{d}$ by substituting (8) into (9),

$$
P_{d}=\frac{A D+B C}{A-C}=g\left(\gamma_{d}\right)
$$

Now we can get the utility function for data users by combining (10) and (5),

$$
U_{d}=\operatorname{Rr} \frac{f\left(\gamma_{d}\right)}{g\left(\gamma_{d}\right)} \quad(\mathrm{b} / \mathrm{J})
$$

Given voice and data user numbers and the spreading gain for data users, we can get the maximal utility and $\gamma_{o p t}$ by differentiating (11) with respect to $\gamma$ and set the derivative to zero. Next, we set the other feasible spreading gains according to WCDMA system parameters and get the corresponding $\gamma_{o p t}$ in the same way. To choose the operation point from these feasible spreading gain and $\gamma_{\text {opt }}$ pairs, we need to see which one can get the maximal throughput using (6). At last, $P_{d}$ and $P_{v}$ can be obtained from (10) and (9) respectively using the operation pair we choose.

\section{PERFORMANCE ANALYSIS AND NUMERICAL RESULTS}

Now we will illustrate the analysis above and performance of the proposed DPGC with simulation results. The system parameters are listed in the next table.

In table 1, the QoS requirements for data and voice traffic are totally different. Data aims for maximal throughput with a lower bound, while voice for acceptable BER.

\begin{tabular}{|l|l|l|l|}
\hline System Parameter & \multicolumn{2}{|l|}{ Voice } & Data \\
\hline Chip Rate & $W$ & $4.096 \times 10^{6}$ chips/s \\
\hline Spreading gain & $G$ & 256 & $\begin{array}{l}\text { 32 or } 64 \text { or } \\
128\end{array}$ \\
\hline Code Rate & $r$ & 0.75 & \\
\hline Code Gain & $\beta$ & 2 & \\
\hline Code index & $k$ & 0.5 & \\
\hline Packet Length & $L$ & 700 bits & \\
\hline QoS:BER & & $\leq 10^{-3}$ & NA \\
\hline $\begin{array}{l}\text { QoS: } \\
\text { Throughput }\end{array}$ & $T$ & NA & $\geq 20$ kbss \\
\hline
\end{tabular}

Table 1. System Parameter 
In this simulation, we must first determine the system capacity, i.e. the users of both kinds of traffic that the system can support in the term of QoS requirement.

Figure 1 presents the system admissible region using DPGC and NAPC [6] with fixed spreading gain 32 . The line represents the limitation condition and the space below the line is the feasible state space, which means all the combination of two kinds of users in the feasible region can be supported by the system and vice versa. It's obvious that the proposed scheme DPGC can support more data users than NAPC, due to the flexibility to ameliorate the MAI. Figure 2 illustrates the data users spreading gain assignment. ' + ' and $\because$ stand for spreading gain 32 and 64 respectively. We can see clearly that when there are fewer users (especially the data users) in the system, it will assign 32 spreading gain to data users for the sake of maximizing the packet throughput. On the other hand, with the increasing of data users, it will assign a larger spreading gain as 64 to data users such that they can have a better packet success rate and therefore more data users can be supported at the same time at the price of lower throughput per user.

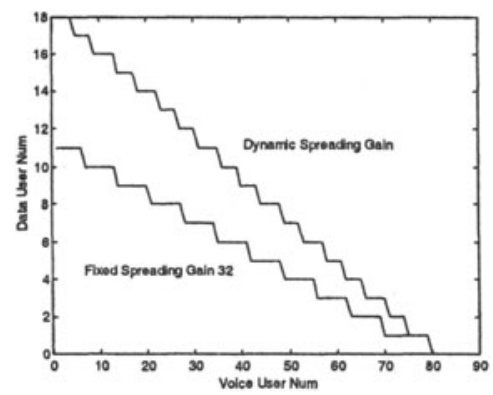

Fig 1. System Admissible Region

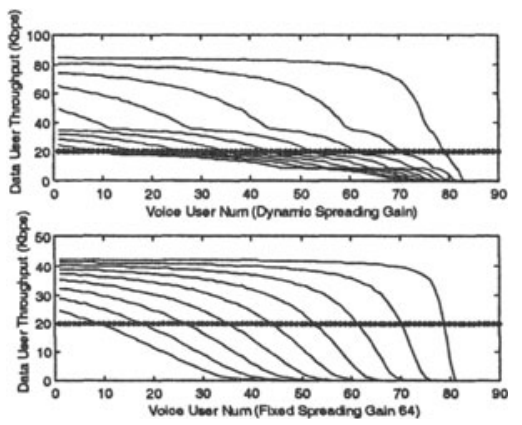

Fig 3.Data User Throughput

(dynamic G vs. fixed G 64)

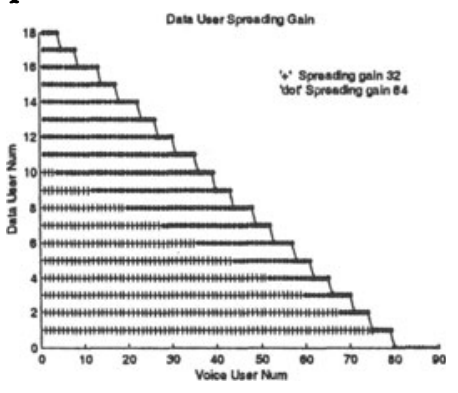

Fig 2. Data User Spreading Gain

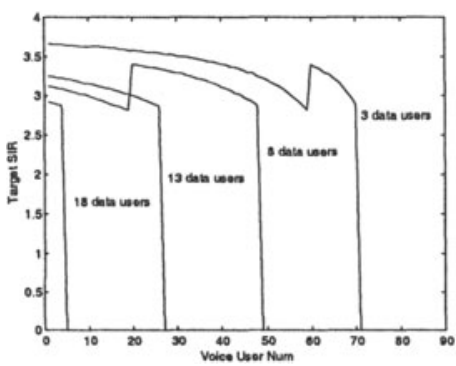

Fig 4. Data User Target SIR 
Figure 3 compares the data users' throughput of DPGC and NAPC with a fixed spreading gain 64 when there are voice users ranging from 1 to 80 . The curves in the figure stand for the throughput of 1 to 18 data users from top to bottom at the step of 2 users. The dashed line is the throughput lower bound for data users. It's obvious that when there are less than 10 users, the throughput of DPGC is much larger than that of NAPC. In case of NAPC with fixed spreading gain 32, it cannot support 18 data users as DPGC. Also note worthy is that the drop of throughput with the increase of data users is much more severe than the increase of voice users.

Unlike voice user, who has a fixed target SIR, data users varies their target SIR to balance the power and throughput. Figure 4 illustrates the target SIR for 3, 8, 13 and 18 data users. The turning points represent the change of spreading gain. Target SIR will influence another performance measure, packet success rate, which will eventually determine the throughput. Figure 5 shows the packet success rate for data users. There exists a direct proportion relationship between target SIR and packet success rate, that's why they have familiar curves. We can point out from this figure that the scheme works well since it can at least obtain a packet success rate of 0.4 .

The power utility ratio of DPGC to NAPC with fixed spreading gain 32 is shown in figure 6. The curves in this figure stand for the ratio of 3, 8, 13 and 18 users respectively from right to left. The bigger the ratio, the better the power efficiency. Therefore, DPGC can obtain a better power efficiency than NAPC by 3000 to 6000 times especially when DPGC supports as many data users as possible.

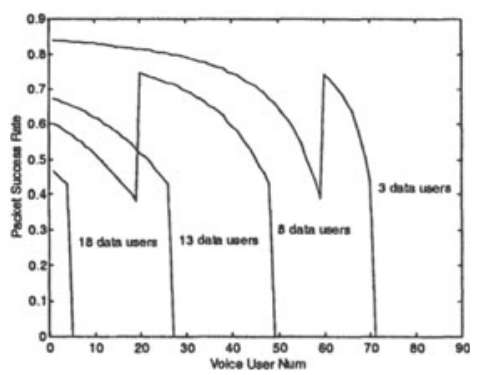

Fig 5. Data User Packet Success Rate

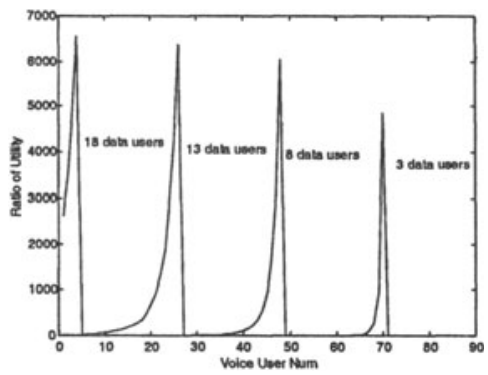

Fig 6. Ratio of Utility (dynamic G to fixed G 32) 


\section{DISCUSSION AND CONCLUSION}

In this paper, we have addressed issues in the support of multimedia services in a wideband CDMA system. In particular, a resource allocation scheme, Dynamic Power and Spreading Gain Control (DPGC), for multimedia wideband CDMA system is presented. The scheme allocates bandwidth and power for data and voice users, and it differentiates voice from data calls by using higher spreading gain. By dynamically changing spreading gain, DPGC can achieve better throughput, system capacity and power utility than pure power control scheme. The simulation results show that the proposed resource allocation scheme DPGC is quite effective in increasing capacity of data users with higher SIR/throughput requirement and lower power constraint. For practical implementation, it only requires the network to broadcast the current user number in the system so that the mobile terminal can look-up for the corresponding spreading gain; while the basestation receiver will update the corresponding target SIR in its uplink power control without having to inform the mobile terminals.

\section{References}

[1] L.C. Yun and D.G. Messerschmitt, "Power control for variable QoS on a CDMA channel," Proc. IEEE MILCOM, vol. 1, Fort Monmouth, NJ, pp.178182, Oct. 1994

[2] C.L.I and K.K. Sabnani, "Variable spreading gain CDMA with adaptive control for integrated traffic in wireless networks," Proc. IEEE VTC, vol.2, Chicago, IL, pp. 794-798, July 1995

[3] Da Rocha Lima, A., Brandao, J.C., "General analysis of downlink power control in CDMA systems," Telecommunications Symposium, 1998. ITS '98 Proceedings. SBT/IEEE International, Vol.1, pp. 172 -176, 1998

[4] J.F.Whitehead, "Signal-level-based dynamic power control for co-channel interference management," in Proc. IEEE Vehicular Technology Conf.,Secaucus,NJ, May 1993, pp. 499-502

[5] S.Ariyavisitakul, "Signal and interference statistics of a CDMA system with feedback power control," IEEE Trans. Commun., Vol. 41, pp. 1626-1634, Nov. 1993

[6] D. Goodman and N. Mandayam, "Network assisted power control for wireless data," VTC 2001 Spring. IEEE VTS 53rd Vol.2, pp.1022-1026,

[7] Hailong Huang, Francois Chin, "Maximizing uplink packet throughput and power efficiency for DS-CDMA based wireless data system", 3Gwireless'2002, May, 2002 
[8] Hailong Huang, Francois Chin, "Performance evaluation of various Network Assisted based Power, Packet and Spreading Gain Control scheme for DSCDMA based wireless data system", VTC2002 Fall

[9] Joon Bae Kim, Michael L.Honig, " Resource allocation for multiple classes of DS-CDMA traffic", IEEE Trans. On Vehicular Technology, vol. 49, pp. 506519, March 2000. 